\title{
Cost-effectiveness of Anbainuo plus methotrexate compared to conventional disease-modifying antirheumatic drugs for rheumatoid arthritis patients in China
}

\author{
Feng Tian", Zhenhua Wen", Jingyang Li, Xiaowen Luo, Li Deng, Liang Zhang, Jingyun He, Fangling Yao, \\ Zheng Liao \\ Department of Rheumatology and Immunology, Zhuzhou Central Hospital, The Affiliated Zhuzhou Hospital Xiangya Medical College CSU, \\ Zhuzhou, China \\ Contributions: (I) Conception and design: J Li; (II) Administrative support: L Deng, L Zhang; (III) Provision of study materials or patients: F Tian, Z \\ Wen, J He, J Li; (IV) Collection and assembly of data: F Tian, Z Wen, X Luo; (V) Data analysis and interpretation: F Tian, Z Wen, F Yao, Z Liao; (VI) \\ Manuscript writing: All authors; (VII) Final approval of manuscript: All authors. \\ "These authors contributed equally to this work. \\ Correspondence to: Jingyang Li, MB. Department of Rheumatology and Immunology, Zhuzhou Central Hospital, The Affiliated Zhuzhou Hospital \\ Xiangya Medical College CSU, 116 South Changjiang Road, Zhuzhou 412007, China. Email: jinyi7139022@163.com.
}

Background: This study aimed to evaluate the cost-effectiveness of Anbainuo (ABN) plus methotrexate (MTX) vs. conventional disease-modifying antirheumatic drugs (cDMARDs) in Chinese rheumatoid arthritis (RA) patients.

Methods: A total of 90 RA patients who underwent ABN + MTX [assigned as ABN + MTX group (n=47)] or cDMARDs [assigned as control group $(\mathrm{n}=43)$ ] treatment were analyzed. Disease activity was assessed at baseline (M0), $3^{\text {rd }}$ month (M3), $6^{\text {th }}$ month (M6), and $12^{\text {th }}$ month (M12) after treatment. Drug, other medical, indirect, and total costs were calculated. Then, pharmacoeconomic analyses were performed with the threshold of cost-effectiveness set as 3 times of the mean gross domestic product (GDP) per capita in China during the study period.

Results: Treatment response rate was similar between the 2 groups, while disease remission and low disease activity (LDA) rates were increased in the ABN + MTX group compared to control group. Drug cost, other medical costs, and total cost were higher in the ABN + MTX group than control group, while indirect cost was similar between the 2 groups. Meanwhile, the quality-adjusted life-years (QALY) in ABN + MTX group and control group were 0.72 and 0.48 years, respectively. The incremental cost-effectiveness ratios (ICER) of ABN + MTX group compared to control group among the entire participant cohort, moderate-diseaseactivity participants, and severe-disease-activity participants were $¥ 135,486.7$, ¥146,450.4, and ¥124,987.2/ QALY, respectively, which were all below the cost-effectiveness threshold. Further sensitivity analyses revealed that the cost-effectiveness of $\mathrm{ABN}+\mathrm{MTX} v s$. cDMARDs was relatively robust, while among all the indexes, ABN price and Health Assessment Questionnaire Disability Index (HAQ-DI) score change for the $\mathrm{ABN}+\mathrm{MTX}$ group affected ICER most.

Conclusions: Treatment with ABN + MTX offers acceptable cost-effectiveness compared to cDMARDs treatment in Chinese RA patients.

Keywords: Anbainuo (ABN); methotrexate (MTX); conventional disease-modifying antirheumatic arthritis drugs; rheumatoid arthritis (RA); cost-effectiveness

Submitted May 25, 2021. Accepted for publication Jul 15, 2021.

doi: 10.21037/atm-21-3132

View this article at: https://dx.doi.org/10.21037/atm-21-3132 


\section{Introduction}

Rheumatoid arthritis (RA) is one of the most common chronic, inflammatory, autoimmune diseases with a prevalence of $0.5-1 \%$ worldwide and $0.19-0.4 \%$ in China $(1,2)$. Clinically, RA patients not only suffer from swelling and pain in small joints (e.g., metacarpophalangeal) and large joints (e.g., ankle), but may also be affected by extra-articular manifestations such as anemia, osteoporosis, interstitial lung disease, cardiovascular disease (3). Meanwhile, RA patients might develop multiple comorbidities (e.g., cardiovascular disease) as a consequence of chronic inflammation (4). Most devastatingly, if insufficiently treated, RA will cause cumulative irreversible joint damage and lead to disability, which greatly affects the quality of life of RA patients and aggravates humanistic as well as economic burden (5).

Tumor necrosis factor (TNF) inhibitors are a vital group of biological disease-modifying antirheumatic drugs (DMARDs), which remarkably suppress the disease progression of RA patients by rapidly ameliorating systematic inflammation, reducing disease activity, suppressing radiology progression to some extent, and greatly improving their quality of life $(6,7)$. However, TNF inhibitors are quite costly, and only a few RA patients in China will readily pay for them due to the relatively low income of Chinese residents and the need for sustained administration of these drugs $(8,9)$. Therefore, the costeffectiveness of TNF inhibitors should be investigated, which may help to guide the administration of TNF inhibitors in RA patients in China.

Anbainuo (ABN), a biosimilar to etanercept (a kind of TNF inhibitor), is a recombinant human TNF- $\alpha$ receptor II: Fc fusion protein developed by a Chinese pharmaceutical company (10). According to a phase II trial, $\mathrm{ABN}+$ methotrexate (MTX) showed increased treatment efficacy compared to ABN or MTX monotherapy in MTXnaïve RA patients (10); another phase III trial revealed that ABN + MTX has good efficacy and tolerance in MTX inadequate-response RA patients compared to placebo (11). Thus, $\mathrm{ABN}$ is a promising biological agent to treat RA patients in China. Moreover, the cost of $A B N$ is far less than original etanercept, which might enable $\mathrm{ABN}$ to become an available and popular TNF inhibitor in RA patients in China. In order to investigate its cost-effectiveness, our small sample-sized pilot study revealed that $\mathrm{ABN}+$ MTX is cost-effective compared to cDMARDs in treating RA patients (12); however, these results require further verification with larger populations and longer follow-up duration to make a solid conclusion regarding this issue. Therefore, subsequently, in this study, we enrolled $55 \mathrm{ABN}$ + MTX-treated patients and 54 cDMARDs-treated patients, and evaluated the treatment efficacy/utility as well as cost during the study, aiming to compare the cost-effectiveness of $\mathrm{ABN}+\mathrm{MTX}$ versus cDMARDs in Chinese RA patients.

We present the following article in accordance with the CHEERS reporting checklist (available at https://dx.doi. org/10.21037/atm-21-3132).

\section{Methods}

\section{Patients}

From March 2015 to October 2018, totally 109 RA patients who underwent $\mathrm{ABN}+\mathrm{MTX}$ or conventional diseasemodifying antirheumatic drugs (cDMARDs) treatment at Zhuzhou Central Hospital were enrolled in this cohort, real-clinical setting, pharmacoeconomic study. The screening criteria referred to the protocol described in our first-stage study (12). This study was approved by the Ethics Committee of Zhuzhou Central Hospital (2015-K05010). All procedures were conducted in accordance with the provisions of the Declaration of Helsinki (as revised in 2013) and Good Clinical Practice guidelines as defined by the International Conference on Harmonization. All participants provided written informed consent. The study was registered on Chinese Clinical Trial Registry (ChiCTR) affiliated to the World Health Organization (WHO) (www. chictr.org.cn) with registry number ChiCTR2000032534.

\section{Data collection}

After enrollment, the baseline (M0) characteristics of participants were recorded, including age, gender, disease duration, tender joint count (TJC), swollen joint count (SJC), C-reactive protein (CRP) level, erythrocyte sedimentation rate (ESR) level, pain visual analogue scale (VAS) score, patient global assessment (PGA) score, physician global assessment (PhGA) score, Health Assessment Questionnaire Disability Index (HAQ-DI) score, and history of treatment. The disease activity of patients was assessed according to the disease activity score with 28 joints based on CRP (DAS28-CRP) and disease activity score with 28 joints based on ESR (DAS28-ESR), which were calculated as follows: DAS28-CRP $=0.56 \times$ $\operatorname{sqrt}($ TJC28) $+0.28 \times \operatorname{sqrt}($ SJC28 $)+0.014 \times \mathrm{PGA}+0.36 \times$ $\ln (\mathrm{CRP}+1)+0.96 ;$ DAS28-ESR $=0.56 \times \operatorname{sqrt}(\mathrm{TJC} 28)+0.28$ $\times \operatorname{sqrt}(\mathrm{SJC} 28)+0.014 \times \mathrm{PGA}+0.70 \times \ln (\mathrm{ESR})$. 


\section{Treatment}

After enrollment, 55 patients about to receive ABN + MTX therapy were assigned to the $\mathrm{ABN}+\mathrm{MTX}$ group, and the other 54 patients who scheduled to receive cDMARDs treatment were allocated to the control group. The treatment regimens for the 2 groups were conducted in accordance with the protocol described in our first-stage study (12). In brief, for the ABN + MTX group, ABN and MTX were administrated to the participants as follows: ABN $25 \mathrm{mg}$ twice a week subcutaneously for 24 weeks or ABN $50 \mathrm{mg}$ once a week subcutaneously for 24 weeks, and MTX 10-20 mg once a week orally for 24 weeks. For the control group, all participants received cDMARDs alone or in combination for 24 weeks, and 23 participants received MTX (10-20 mg once a week orally) + leflunomide [10 mg once a day (qd)], 31 patients received MTX (10-20 mg once a week orally) + sulfasalazine [1 g twice a day (tid) orally $]+$ hydroxychloroquine (400 mg qd).

\section{Follow-up}

After initiation of treatment, regular participant follow-up was conducted, and the TJC, SJC, ESR level, CRP level, pain VAS score, PGA score, PhGA score, and HAQ-DI score were evaluated at the $3^{\text {rd }}$ month (M3), $6^{\text {th }}$ month (M6), and $12^{\text {th }}$ month (M12). During follow-up, 13 participants lost follow-up without any assessment, 6 withdrew their informed consents, and those 19 patients in total were excluded from the final analysis. Therefore, 90 participants were included in the final analysis: 47 cases in the $A B N+$ MTX group and 43 cases in the control group.

\section{Efficacy assessment}

The primary outcome of efficacy was DAS28-ESR remission rate, defined as the percentage of participants with DAS28-ESR $\leq 2.6$ after treatment. The secondary outcome of efficacy included DAS28-ESR response rate (defined as the percentage of patients with an improvement of DAS28-ESR >1.2 after treatment), DAS28-ESR low disease activity (LDA) rate (defined as the percentage of patients with DAS28-ESR $\leq 3.2$ after treatment), and the improvements of TJC, SJC, CRP level, ESR level, pain VAS score, PGA score, PhGA score, HAQ-DI score, DAS28-CRP score, and DAS28-ESR score. The DAS28ESR remission rate, DAS28-ESR response rate, and DAS28-ESR LDA rate were assessed at M6 and M12. The changes of TJC, SJC, CRP level, ESR level, pain VAS score, PGA score, PhGA score, HAQ-DI score, DAS28CRP score, and DAS28-ESR score was calculated at M3, M6, and M12.

\section{Direct, indirect, and total costs calculation}

Direct costs included drug costs and other medical costs. Drug costs for participants were calculated according to unit cost and the dosages recorded in the case report form (CRF). Other medical costs included outpatient cost, emergency cost, and hospitalization cost. Indirect costs included lost productivity costs of participants and their caregivers due to working days lost, calculated by the lost working days multiplied by the local average daily wage. Total costs were defined as the sum of drug costs, other medical costs, and indirect costs. All unit costs of medication, outpatient, emergency, and hospitalization were obtained from the electronic medical records of Zhuzhou Central Hospital.

\section{Pharmacoeconomic assessment}

Cost-effectiveness analysis was used for pharmacoeconomic assessment. The total costs and quality-adjusted life-year (QALY) was calculated, and the difference of incremental cost-effectiveness ratio (ICER) between 2 groups was evaluated by incremental cost divided by incremental QALY (12). The QALY was defined according to the study we published previously (12), which was estimated from a relation function between HAQ-DI scores and the Europe Quality of Life 5-dimension (EQ-5D) questionnaire utility values (13): EQ-5D $=0.9567-0.309 \times \mathrm{HAQ}-\mathrm{DI}$. The threshold of acceptable cost-effectiveness was an ICER lower than 3 times of the annual gross domestic product (GDP) per capita, which was defined with reference to the willingness-to-pay (WTP) recommended by the WHO Choosing Interventions that are Cost-Effective (CHOICE) program (14). In the case of China, GDP per capita was 53,980 RMB (¥) in $2016, ¥ 59,660$ in $2017, ¥ 66,006$ in 2018 , $¥ 70,892$ in 2019 , and the average of GDP per capita from 2016 to 2019 was $¥ 62,634$.

\section{Sensitivity analyses}

To evaluate the uncertainty of market effects on direct or indirect medical costs, the drug price $(\mathrm{ABN})$, other medical cost, and indirect cost were varied in sensitivity analyses as follows: (I) fell by $20 \%$ and $50 \%$ of their base-case prices; 
(II) rose by $20 \%$ and $50 \%$ of their base-case prices. Besides, the uncertainty of improvement for HAQ-DI score on costeffectiveness was also assessed by the sensitivity analyses, which were carried out as follows: (I) fell by $20 \%$ and $50 \%$ of HAQ-DI score in ABN + MTX group; (II) rose by $20 \%$ and $50 \%$ of HAQ-DI score in ABN + MTX group.

\section{Statistical analysis}

Statistical analysis was performed using the statistical software SPSS 21.0 (IBM Corp., Armonk, NY, USA), and figures were made with the use of GraphPad Prism 6.01 software (GraphPad Software Inc., La Jolla, CA, USA). Data were presented as mean \pm standard deviation (SD) or count (percentage). Comparison between 2 groups was determined by Student's $t$-test or chi-square test. All tests were 2 -sided, and a $\mathrm{P}$ value $<0.05$ was considered statistically significant.

\section{Results}

\section{Study flow}

A total of $211 \mathrm{RA}$ patients were invited to take part in our study, 48 of whom were excluded (25 patients who missed the invitation, 15 who did not agree to participate, and 8 who were unable to participate due to distance). The remaining 163 RA patients were screened for eligibility, among whom 54 either did not meet the inclusion criteria or met the exclusion criteria and were thus excluded from this study. Therefore, 109 RA patients were enrolled and assigned to either the ABN + MTX group or control group. In the ABN + MTX group ( $\mathrm{n}=55$ ), participants received $\mathrm{ABN}+\mathrm{MTX}$ for 24 weeks and were subsequently followedup for 12 months after initiation of their treatment. During the follow-up period, 8 participants were excluded (6 participants who were lost to follow-up without any assessment and 2 who withdrew their informed consent); finally, 47 RA participants in the ABN + MTX group were included in the efficacy and pharmacoeconomic analyses. In the control group $(\mathrm{n}=54)$, participants received cDMARDs for 24 weeks and were also followed-up for 12 months after initiation of their treatment. During the follow-up period, 11 participants were excluded (7 participants who were lost to follow-up without any assessment and 4 who withdrew their informed consent); finally, 43 RA participants in the control group were included in the efficacy and pharmacoeconomic analyses (Figure 1).

\section{Participant baseline clinical characteristics}

The mean age of RA patients was $55.5 \pm 13.0$ years in the $\mathrm{ABN}+\mathrm{MTX}$ group and $58.3 \pm 8.6$ years in the control group. There were 37 (78.7\%) females and $10(21.3 \%)$ males in the ABN + MTX group, and 35 (81.4\%) females and $8(18.6 \%)$ males in the control group. The mean disease duration was $5.2 \pm 3.3$ years in the ABN + MTX group and $4.9 \pm 2.4$ years in the control group. The mean CRP, ESR, and DAS28-ESR scores were $39.5 \pm 39.1 \mathrm{mg} / \mathrm{L}$, $60.4 \pm 34.5 \mathrm{~mm} / \mathrm{h}$, and $5.3 \pm 0.9$, respectively, in the $\mathrm{ABN}+$ MTX group, and $42.8 \pm 31.6 \mathrm{mg} / \mathrm{L}, 62.6 \pm 37.9 \mathrm{~mm} / \mathrm{h}$, and $5.1 \pm 1.0$, respectively, in the control group. The numbers of participants with moderate and severe disease activity were $15(31.9 \%)$ and $32(68.1 \%)$ in the ABN + MTX group, and $18(41.9 \%)$ as well as $25(58.1 \%)$ in the control group, respectively. The detailed baseline characteristics are shown in Table 1.

\section{Treatment efficacy}

The change of DAS28-ESR (Figure 2A), DAS28-CRP (Figure 2B), TJC (Figure 2C), SJC (Figure 2D), pain VAS (Figure 2E), PGA (Figure 2F), PhGA (Figure 2G), and HAQ-DI (Figure $2 H$ ) were more obvious in the ABN + MTX group compared to the control group at several time points (all $\mathrm{P}<0.05)$. However, changes in ESR (Figure $2 I$ ) or CRP (Figure 2f) were similar between the 2 groups at each visit (all $\mathrm{P}>0.05$ ). Moreover, for DAS28-ESR response rate, no difference was found between the ABN + MTX group and the control group at M6 $(\mathrm{P}=0.101)$ or M12 $(\mathrm{P}=0.194)$ (Figure 3A). Both DAS28-ESR remission rate and DAS28ESR LDA rate were increased in the ABN + MTX group compared to the control group at M12 (both $\mathrm{P}<0.01$ ), but not at M6 (both $\mathrm{P}>0.05$ ) (Figure 3B,C).

\section{Direct, indirect, and total costs}

Drug cost was increased in the ABN + MTX group

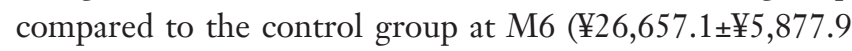
vs. $¥ 3,097.2 \pm ¥ 1,786.1)$ and M12 (¥38,433.3 $¥ 14,696.6$ vs. $¥ 9,964.3 \pm ¥ 2,281.9$ ) (both $\mathrm{P}<0.001)$. Other medical cost was also greater in the ABN + MTX group compared to the control group at M6 (¥11,036.4 $¥ 7,833.5 \mathrm{vs}$. $¥ 7,980.0 \pm ¥ 2,233.8)(\mathrm{P}=0.013)$ and $\mathrm{M} 12(¥ 18,306.1 \pm ¥ 13,726.5$ vs. $¥ 13,641.1 \pm ¥ 1,281.1)(\mathrm{P}=0.025)$. Indirect cost was similar between the 2 groups at M6 $(¥ 5,184.3 \pm ¥ 5,753.5$

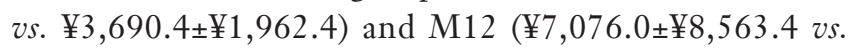




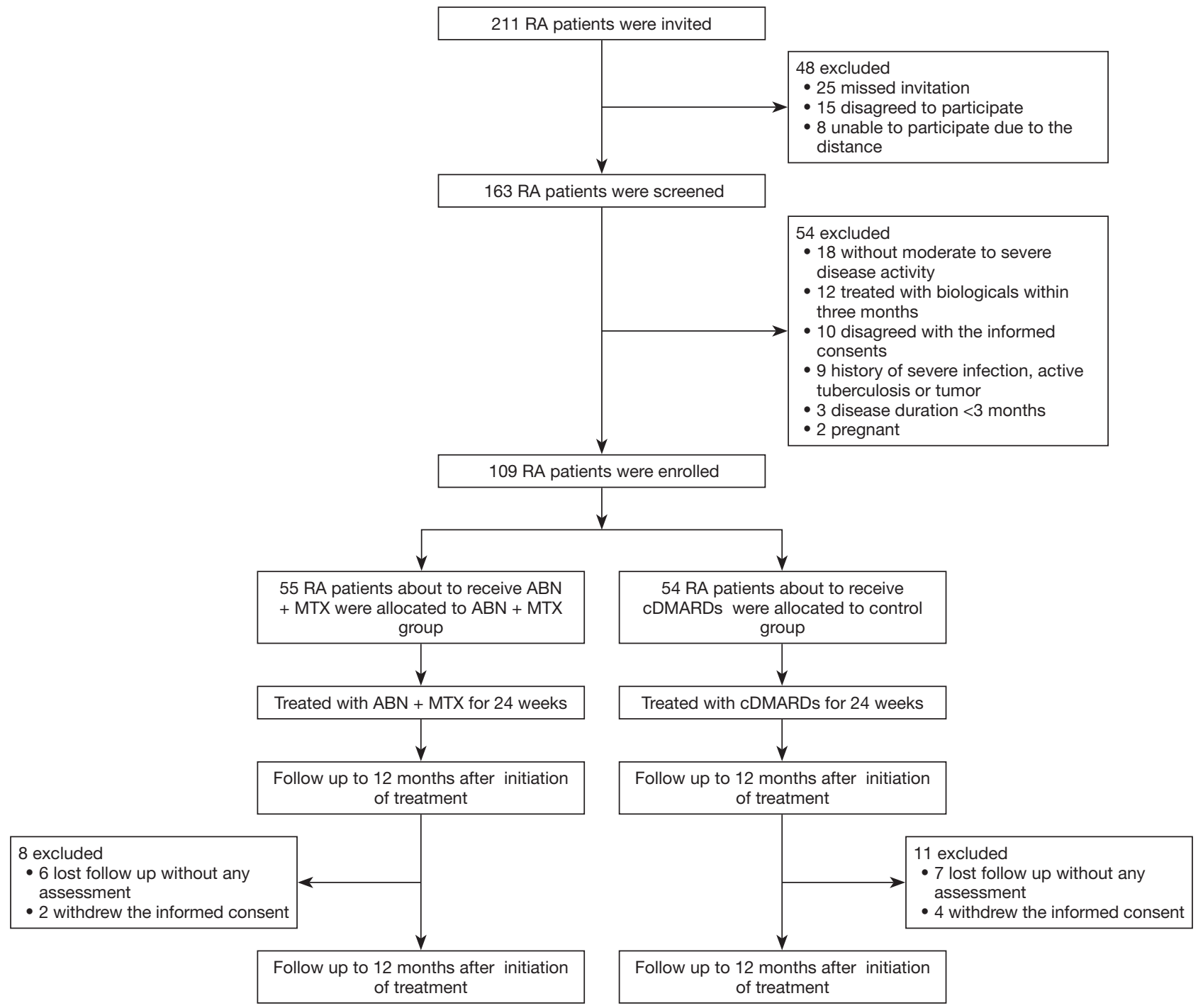

Figure 1 Study flow chart. RA, rheumatoid arthritis; ABN, Anbainuo; MTX, methotrexate; cDMARDs, conventional disease-modifying antirheumatic drugs.

$¥ 7,693.2 \pm ¥ 732.1$ ) (both $\mathrm{P}>0.05$ ). After calculation, the total cost was higher in the ABN + MTX group than the control group at M6 (¥42,877.8 $¥ 13,652.3$ vs. $¥ 14,767.7 \pm ¥ 3,276.1)$ and M12 ( $¥ 63,815.4 \pm ¥ 26,520.6$ vs. $¥ 31,298.6 \pm ¥ 2,821.3$ ) (both $\mathrm{P}<0.001)$ (Table 2).

\section{Cost-effectiveness}

The ABN + MTX group and control group had QALY of 0.72 years and 0.48 years, respectively, thus the $\mathrm{ABN}+$ MTX group achieved an incremental QALY of 0.24 years compared to the control group. Total cost in the ABN + MTX group and control group was $¥ 63,815$.4 and $¥ 31,298$.6, respectively, resulting in an incremental cost of $¥ 32,516.8$ in ABN + MTX group compared to control group. Based on the above data, the ABN + MTX group had an ICER of $¥ 135,486.7$ per QALY compared to the control group, which was less than 3 times the mean GDP per capita in China during the study period (Table 3). Therefore, ABN + MTX treatment showed an acceptable cost-effectiveness over cDMARDs treatment in RA patients. Moreover, costeffectiveness analysis in subgroups was further performed. Data showed that in moderate RA patients, the ABN + MTX group generated an incremental QALY of 0.24 years and an incremental cost of $¥ 35,148.1$, resulting in an ICER of $¥ 146,450.4$ per QALY compared to the control 
Table 1 Baseline characteristics

\begin{tabular}{|c|c|c|c|}
\hline Items & $A B N+M T X$ group $(N=47)$ & Control group (N=43) & $P$ value \\
\hline Gender, No. (\%) & & & 0.752 \\
\hline Female & $37(78.7)$ & $35(81.4)$ & \\
\hline Male & $10(21.3)$ & $8(18.6)$ & \\
\hline Disease activity, No. (\%) & & & 0.328 \\
\hline Moderate & $15(31.9)$ & $18(41.9)$ & \\
\hline Severe & $32(68.1)$ & $25(58.1)$ & \\
\hline TJC, mean \pm SD & $10.0 \pm 5.5$ & $9.3 \pm 5.5$ & 0.588 \\
\hline $\mathrm{ESR}(\mathrm{mm} / \mathrm{h})$, mean $\pm \mathrm{SD}$ & $60.4 \pm 34.5$ & $62.6 \pm 37.9$ & 0.772 \\
\hline Pain VAS score, mean \pm SD & $6.9 \pm 1.9$ & $7.0 \pm 1.5$ & 0.865 \\
\hline $\mathrm{PGA}$ score, mean $\pm \mathrm{SD}$ & $6.9 \pm 2.0$ & $7.3 \pm 1.3$ & 0.331 \\
\hline PhGA score, mean \pm SD & $6.7 \pm 1.9$ & $7.1 \pm 1.3$ & 0.246 \\
\hline $\mathrm{HAQ}$-DI score, mean $\pm \mathrm{SD}$ & $1.6 \pm 0.6$ & $1.8 \pm 0.5$ & 0.199 \\
\hline DAS28-CRP score, mean \pm SD & $4.6 \pm 0.9$ & $4.6 \pm 0.9$ & 0.776 \\
\hline DAS28-ESR score, mean \pm SD & $5.3 \pm 0.9$ & $5.1 \pm 1.0$ & 0.518 \\
\hline \multicolumn{4}{|l|}{ History of treatment, No. (\%) } \\
\hline
\end{tabular}

Comparison was determined by Student's t-test or Chi-square test. ABN, anbainuo; MTX, methotrexate; SD, standard deviation; TJC, tender joint count; SJC, swollen joint count; CRP, C-reactive protein; ESR, erythrocyte sedimentation rate; VAS, visual analogue scale; PGA, patient global assessment; PhGA, physician global assessment; HAQ-DI, Health Assessment Questionnaire Disability Index; DAS28CRP, disease activity score with 28 joints based on CRP; DAS28-ESR, disease activity score with 28 joints based on ESR; cDMARDs, conventional disease-modifying antirheumatic drugs; NSAIDs, non-steroidal anti-inflammatory drugs.

group, which was less than 3 times the mean GDP per capita in China. Meanwhile in severe RA patients, ABN + MTX group had an incremental QALY of 0.25 years and an incremental cost of $¥ 31,246.8$, thus yielding an ICER of $¥ 124,987.2$ per QALY compared to the control group, which was less than 2 times the mean GDP per capita in China during the study period (Table 4). Therefore, compared to cDMARDs treatment, ABN + MTX treatment presented acceptable cost-effectiveness in moderate RA patients, and good cost-effectiveness in severe RA patients.

\section{Sensitivity analysis}

The robustness of the cost-effectiveness results was tested by sensitivity analysis, in which the main indexes were decreased or increased by $20 \%$ or $50 \%$. The resulting data showed that: (I) increasing $\mathrm{ABN}$ price by $20 \%$, decreasing ABN price by $20 \%$ and $50 \%$ yielded an ICER of $¥ 163,632.1$, $¥ 105,556.7$, and $¥ 54,309.2$ per QALY, respectively, in $\mathrm{ABN}+\mathrm{MTX}$ group vs. control group, which was less than 3 times, 2 times, and 1 time the mean GDP per capita in China, respectively; whereas increasing $\mathrm{ABN}$ price by $50 \%$ 


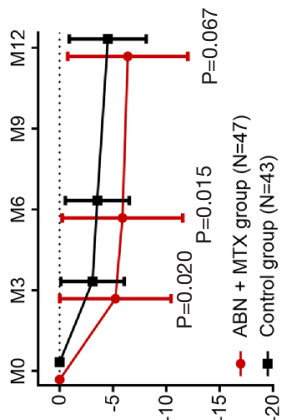

ow moג כrs to əธิueบว

$\square$

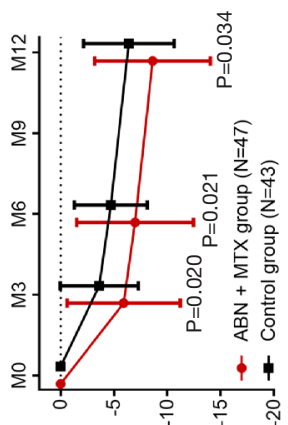

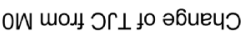

U

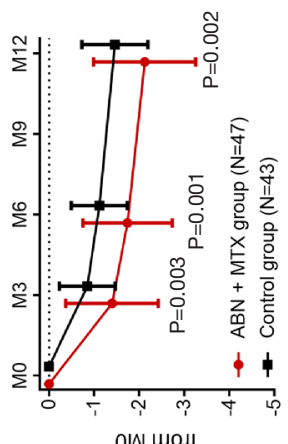

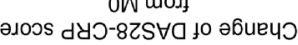

๓

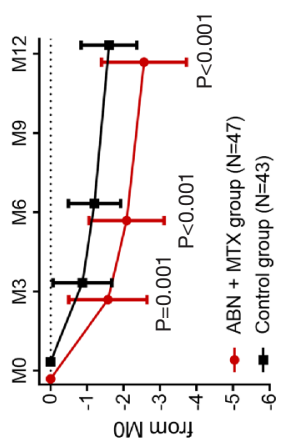

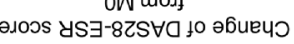

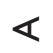

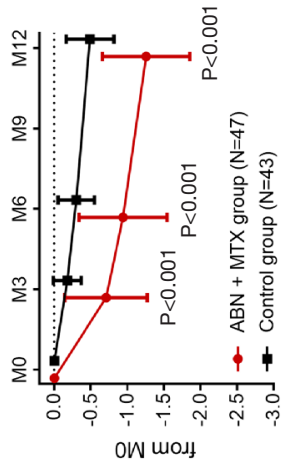

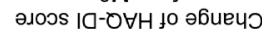

I
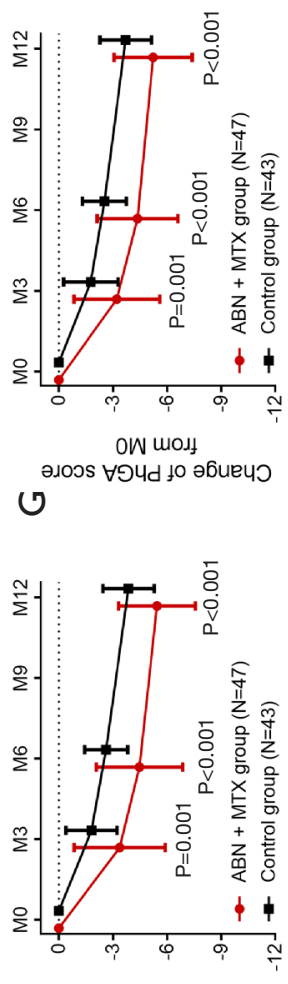

OW modt
әлоos $\forall$ Od to ә6ueyว

ㄴ.

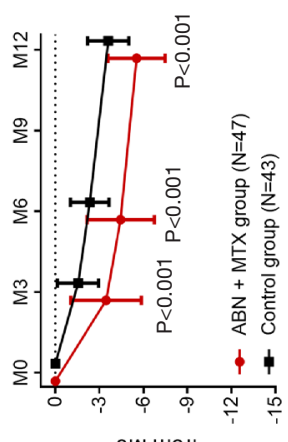

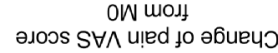
山
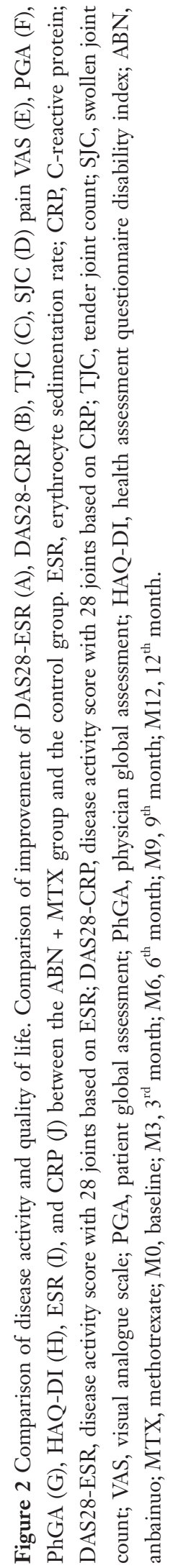

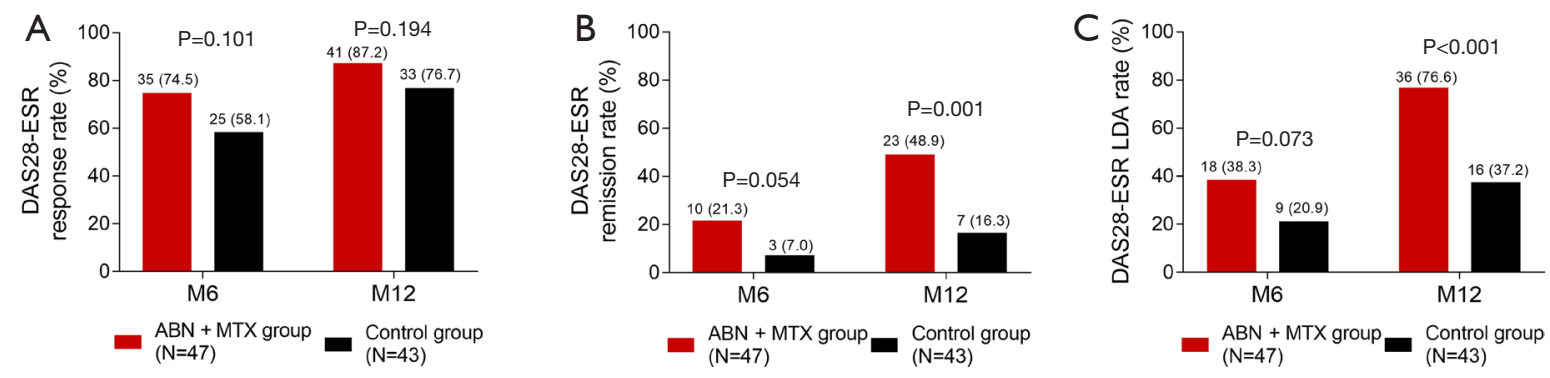

Figure 3 Comparison of treatment efficacy. DAS28-ESR response rate (A), DAS28-ESR remission rate (B), DAS28-ESR LDA rate (C) between the ABN + MTX group and the control group. DAS28-ESR, disease activity score with 28 joints based on erythrocyte sedimentation rate; LDA, low disease activity; ABN, anbainuo; MTX, methotrexate; M6, $6^{\text {th }}$ month; M12, $12^{\text {th }}$ month.

Table 2 Comparison of cost between ABN + MTX group and control group

\begin{tabular}{|c|c|c|c|c|c|c|}
\hline Items & \multicolumn{3}{|c|}{ M6 } & \multicolumn{3}{|c|}{ M12 } \\
\hline Drug cost ( $¥)$ & $26,657.1 \pm 5,877.9$ & $3,097.2 \pm 1,786.1$ & $<0.001$ & $38,433.3 \pm 14,696.6$ & $9,964.3 \pm 2,281.9$ & $<0.001$ \\
\hline Other medical cost $(¥)$ & $11,036.4 \pm 7,833.5$ & $7,980.0 \pm 2,233.8$ & 0.013 & $18,306.1 \pm 13,726.5$ & $13,641.1 \pm 1,281.1$ & 0.025 \\
\hline Indirect cost ( $¥)$ & $5,184.3 \pm 5,753.5$ & $3,690.4 \pm 1,962.4$ & 0.099 & $7,076.0 \pm 8,563.4$ & $7,693.2 \pm 732.1$ & 0.625 \\
\hline
\end{tabular}

Comparison was determined by Student's $t$-test. ABN, anbainuo; MTX, methotrexate.

Table 3 Cost-effectiveness analysis

\begin{tabular}{lccccc}
\hline Group & QALY & Incremental QALY & Total cost $(¥)$ & Incremental cost $(¥)$ & ICER $(¥ /$ QALY) \\
\hline ABN + MTX & 0.72 & 0.24 & $63,815.4$ & $32,516.8$ & $135,486.7^{\star}$ \\
Control & 0.48 & - & $31,298.6$ & - & - \\
\hline
\end{tabular}

The average of gross domestic product (GDP) per capita in China from 2016 to 2019 was $¥ 62,634$. ${ }^{*}$, the ICER was less than 3 times of GDP per capita. QALY, quality-adjusted life-years; ICER, incremental cost-effectiveness ratio; ABN, anbainuo; MTX, methotrexate.

Table 4 Cost-effectiveness analysis of subgroup

\begin{tabular}{|c|c|c|c|c|c|c|}
\hline Disease activity & Group & QALY & Incremental QALY & Total cost (¥) & Incremental cost $(¥)$ & ICER (¥/QALY) \\
\hline Moderate & Control & 0.53 & - & $32,347.4$ & & \\
\hline \multirow[t]{2}{*}{ Severe } & $A B N+M T X$ & 0.70 & 0.25 & $62,090.3$ & $31,246.8$ & $124,987.2^{\star \star}$ \\
\hline & Control & 0.45 & - & $30,843.5$ & & \\
\hline
\end{tabular}

The average of gross domestic product (GDP) per capita in China from 2016 to 2019 was $¥ 62,634$. *, the ICER was less than 3 times of the mean GDP per capita; ${ }^{\star \star}$, the ICER was less than 2 times of the mean GDP per capita. QALY, quality-adjusted life-years; ICER, incremental cost-effectiveness ratio; ABN, anbainuo; MTX, methotrexate.

resulted in an ICER of $¥ 199,487.9$ per QALY in ABN + MTX group vs. control group, which exceeded 3 times the mean GDP per capita in China. (II) Increasing HAQ-DI score by $20 \%$, reducing HAQ-DI score by $20 \%$ and $50 \%$ yielded an ICER of $¥ 171,141.1$, $¥ 116,131.4$, and $¥ 92,905.1$ per QALY, respectively, in ABN + MTX group vs. control 
group, which was less than 3 times, 2 times, and 2 times the mean GDP per capita in China, respectively; while promoting HAQ-DI score by $50 \%$ resulted in an ICER of $¥ 250,129.2$ per QALY in ABN + MTX group vs. control group, which exceeded 3 times the mean GDP per capita in China. (III) Regarding other medical cost and indirect cost, increasing or decreasing these 2 indexes by $20 \%$ or $50 \%$ all yielded ICERs that did not exceed 3 times the mean GDP per capita in China. These data indicated that $\mathrm{ABN}$ price and HAQ-DI score, but not other medical cost or indirect cost, impacted the cost-effectiveness of $\mathrm{ABN}+\mathrm{MTX}$ treatment $v$ s. cDMARDs treatment greatly, and ABN + MTX treatment generally exhibited superior cost-effectiveness to cDMARDs treatment in RA patients in China (Table 5). In addition, we used different utility formulae to calculate cost-effectiveness, and found that $A B N+$ MTX treatment was cost-effective than cDMARDs treatment based on all these formulas (Table S1) (13,15-18).

\section{Discussion}

In the present study, we found that: (I) compared to cDMARDs treatment, ABN + MTX treatment showed acceptable cost-effectiveness in RA patients, and subgroup analysis revealed that it offered acceptable cost-effectiveness in moderate RA patients as well as good cost-effectiveness in severe RA patients; (II) the cost-effectiveness of ABN + MTX treatment compared to cDMARDs treatment presented relative robustness to the turbulence of $\mathrm{ABN}$ price and HAQ-DI score.

Since the appearance of TNF inhibitors on the market, the prognosis of RA patients has been considerably improved (6). These TNF inhibitors present great efficacy in controlling disease progression by suppressing

Table 5 Sensitivity analysis

\begin{tabular}{|c|c|c|c|c|c|}
\hline Items & QALY & Incremental QALY & Total cost (¥) & Incremental cost $(¥)$ & ICER ( $¥ / Q A L Y)$ \\
\hline \multicolumn{6}{|l|}{ Drug price } \\
\hline Price of $A B N$ up by $20 \%$ & & & & & $163,632.1^{\star}$ \\
\hline$A B N+M T X$ & 0.72 & 0.24 & $70,570.3$ & $39,271.7$ & \\
\hline Control & 0.48 & - & $31,298.6$ & - & \\
\hline Price of $A B N$ up by $50 \%$ & & & & & $199,487.9$ \\
\hline$A B N+M T X$ & 0.72 & 0.24 & $79,175.7$ & $47,877.1$ & \\
\hline Control & 0.48 & - & $31,298.6$ & - & \\
\hline Price of $A B N$ down by $20 \%$ & & & & & $105,556.7^{\star \star}$ \\
\hline$A B N+M T X$ & 0.72 & 0.24 & $56,632.2$ & $25,333.6$ & \\
\hline Control & 0.48 & - & $31,298.6$ & - & \\
\hline Price of $A B N$ down by $50 \%$ & & & & & $54,309.2^{\star \star \star}$ \\
\hline$A B N+M T X$ & 0.72 & 0.24 & $44,332.8$ & $13,034.2$ & \\
\hline Control & 0.48 & - & $31,298.6$ & - & \\
\hline \multicolumn{6}{|l|}{ Other medical cost } \\
\hline Other medical cost up by $20 \%$ & & & & & $139,374.2^{*}$ \\
\hline$A B N+M T X$ & 0.72 & 0.24 & $67,476.6$ & $33,449.8$ & \\
\hline Control & 0.48 & - & $34,026.8$ & - & \\
\hline Other medical cost up by $50 \%$ & & & & & $145,205.5^{\star}$ \\
\hline$A B N+M T X$ & 0.72 & 0.24 & $72,968.5$ & $34,849.3$ & \\
\hline Control & 0.48 & - & $38,119.2$ & - & \\
\hline
\end{tabular}

Table 5 (continued) 
Table 5 (continued)

\begin{tabular}{|c|c|c|c|c|c|}
\hline Items & QALY & Incremental QALY & Total cost $(¥)$ & Incremental cost ( $¥)$ & ICER (¥/QALY) \\
\hline$A B N+M T X$ & 0.72 & 0.24 & $60,154.2$ & $31,583.8$ & \\
\hline Control & 0.48 & - & $28,570.4$ & - & \\
\hline Other medical cost down by $50 \%$ & & & & & $125,767.5^{\star}$ \\
\hline Control & 0.48 & - & $24,478.1$ & - & \\
\hline \multicolumn{6}{|l|}{ Indirect cost } \\
\hline Indirect cost up by $20 \%$ & & & & & $134,972.1^{*}$ \\
\hline$A B N+M T X$ & 0.72 & 0.24 & $65,230.6$ & $32,393.3$ & \\
\hline$A B N+M T X$ & 0.72 & 0.24 & $67,353.4$ & $32,208.2$ & \\
\hline Control & 0.48 & - & $35,145.2$ & - & \\
\hline Indirect cost down by $20 \%$ & & & & & $136,000.8^{*}$ \\
\hline$A B N+M T X$ & 0.72 & 0.24 & $62,400.2$ & $32,640.2$ & \\
\hline Control & 0.48 & - & $29,760.0$ & - & \\
\hline Indirect cost down by $50 \%$ & & & & & $136,772.5^{\star}$ \\
\hline$A B N+M T X$ & 0.72 & 0.24 & $60,277.4$ & $32,825.4$ & \\
\hline Control & 0.48 & - & $27,452.0$ & - & \\
\hline$A B N+M T X$ & 0.61 & 0.13 & $63,815.4$ & $32,516.8$ & $250,129.2$ \\
\hline Control & 0.48 & - & $31,298.6$ & - & \\
\hline \multicolumn{6}{|l|}{ HAQ-DI score down by $20 \%$} \\
\hline $\mathrm{ABN}+\mathrm{MTX}$ & 0.76 & 0.28 & $63,815.4$ & $32,516.8$ & $116,131.4^{\star \star}$ \\
\hline Control & 0.48 & - & $31,298.6$ & - & - \\
\hline \multicolumn{6}{|l|}{ HAQ-DI score down by $50 \%$} \\
\hline$A B N+M T X$ & 0.83 & 0.35 & $63,815.4$ & $32,516.8$ & $92,905.1^{\star \star}$ \\
\hline Control & 0.48 & - & $31,298.6$ & - & - \\
\hline
\end{tabular}

The average of gross domestic product (GDP) per capita in China from 2016 to 2019 was $¥ 62,634$. *, the ICER was less than 3 times of the mean GDP per capita; ${ }^{*}$, the ICER was less than 2 times of the mean GDP per capita; ${ }^{\star \star *}$, the ICER was less than 1 times of the mean GDP per capita. QALY, quality-adjusted life-years; ICER, incremental cost-effectiveness ratio; ABN, anbainuo; MTX, methotrexate; HAQDI, Health Assessment Questionnaire Disability Index. 
inflammation and disease activity, reducing radiology progression to some extent, as well as improving the quality of life of RA patients (7). However, TNF inhibitors are quite costly. It is estimated that the annual drug cost of TNF inhibitors reaches around $\$ 36,000$ per individual in the United States (3). Moreover, according to a previous study in Poland, the average drug cost for patients who received etanercept for 24 months was 68,514 PLN (19); therefore, the cost-effectiveness of TNF inhibitors should be carefully assessed to support the guidance of TNF inhibitors administration in RA patients. Regarding the cost-effectiveness of TNF inhibitors, a previous study in Finland found that TNF inhibitors are more cost-effective compared with rituximab, a CD20 monoclonal antibody, in RA patients (20). Another study in Germany showed that adalimumab (a TNF inhibitor) is cost-effective compared to cDMARDs in RA patients (21). However, most of these studies have been performed in developed countries, and assessments of cost-effectiveness of TNF inhibitors in Chinese RA patients are relatively deficient and urgently needed.

The drug ABN is a novel biosimilar to etanercept independently developed by a Chinese pharmaceutical company. A phase II trial showed that after the combined administration of ABN and MTX for 24 weeks, the proportions of patients who achieved ACR50 and ACR70 were $53.6 \%$ and $27.7 \%$, respectively, which were significantly higher than monotherapy of $A B N$ or MTX (10). Another phase III trial revealed that the ABN + MTX administration for 12 weeks promoted ACR20, ACR50, and ACR70 rate in MTX-inadequate response RA patients compared to placebo; meanwhile $\mathrm{ABN}$ was welltolerated in those patients (11). Considering that $\mathrm{ABN}$ presents good treatment efficacy as well as lower price than original etanercept, it may be vital to evaluate the cost of ABN + MTX vs. cDMARDs, further investigating their comparison of cost-effectiveness. In the present study, ABN + MTX treatment generated higher drug cost and other medical cost than cDMARDs treatment, resulting in a higher total cost; meanwhile, drug cost (mainly cost of $\mathrm{ABN}$ ) was the dominating cost. Moreover, we found that indirect cost in the ABN + MTX group was numerically lower than that in the control group at M12, which could be explained by the finding that: ABN + MTX had better treatment effect than cDMARDs in RA patients (as mentioned above), which might improve the patient quality of life, thus saving the cost caused by loss of productivity of patients or their caregivers due to loss of working days. These data indicated that ABN + MTX treatment was more costly compared to cDMARDs treatment, and evaluation of the cost-effectiveness of ABN + MTX treatment $v s$. cDMARDs treatment was quite important.

Evaluation of the cost-effectiveness of TNF inhibitors is quite widespread in developed countries, whereas it is relatively lacking in China where only a few such studies have previously been conducted. A previous study revealed that etanercept + MTX treatment was not costeffective compared to triple cDMARDs treatment with an ICER of $¥ 2,939,506.7$ per QALY in treating Chinese RA patients (22). Meanwhile, our pilot study showed that ABN + MTX treatment offered acceptable cost-effectiveness compared to cDMARDs treatment in a small-samplesized Chinese RA participant cohort (12). In order to further verify the results of our pilot study, we conducted the present study and found that the ICER of ABN + MTX treatment compared to cDMARDs treatment was $¥ 135,486.7$ per QALY, which was less than the costeffectiveness threshold. Notably, the cost-effectiveness of $\mathrm{ABN}+\mathrm{MTX}$ treatment compared to cDMARDs treatment was more obvious in severe RA patients, which was less than 2 times the mean GDP per capita in China. Possible explanations might be that: (I) the therapeutic efficacy of $\mathrm{ABN}+\mathrm{MTX}$ treatment was better in severe RA patients, which might cause an increased incremental QALY and reduced ICER; (II) the good treatment effect of ABN + MTX reduced disease activity in severe RA patients, which might decrease the hospitalization cost, thus reducing the total cost in patients who received ABN + MTX. Therefore, $\mathrm{ABN}+\mathrm{MTX}$ treatment represented cost-effectiveness that was more obvious in severe RA patients, and it might be more plausible to administrate ABN + MTX in severe RA patients.

The sensitivity analysis in this study revealed that $\mathrm{ABN}$ cost and HAQ-DI score in the ABN + MTX group were 2 predominant factors that affected the cost-effectiveness of ABN + MTX treatment vs. cDMARDs treatment. Possible explanations might be that: ABN price was much higher than other medical cost and indirect cost (as shown in the cost analysis), thus $\mathrm{ABN}$ price greatly affected total cost and predominantly impacted the cost-effectiveness of ABN + MTX treatment vs. cDMARDs treatment (2). HAQDI score was a dominating factor that impacts the QALY, thus it also greatly affected the cost-effectiveness of ABN + MTX treatment $v s$. cDMARDs treatment. Furthermore, increasing the price of $\mathrm{ABN}$ or reducing HAQ-DI score 
in the ABN + MTX group by $20 \%$ still showed acceptable cost-effectiveness. These data indicated that ABN + MTX treatment $v s$. cDMARDs treatment remained acceptably cost-effective under the premise of the turbulence of $A B N$ price and HAQ-DI score in RA patients (3).

It could be addressed that ultrasound examination was performed in the studied patients. Ultrasound is a recommended method for monitoring synovitis in recent years, since it's more sensitive for disease evaluation compared to traditional symptomatic synovitis, and could predict disease relapse risk. Meanwhile, it's considered that ultrasound synovitis remission (disappearance) is an indicator for dose-tapering treatment, which could reduce the cost. Besides, this study did not evaluate the costeffectiveness of cardiovascular screening; then we carefully searched related articles, which observed that a previous study reports that: screening for cardiovascular events in RA patients is estimated to be cost-effective with high chances of being less expensive and more effective (23).

There were several limitations to this study. Firstly, due to insufficient manpower and funding, we did not perform a patients' survey to investigate the acceptability curve analysis and evaluate the cost-effectiveness within a range of cost-effectiveness thresholds. Instead, the cost-effectiveness threshold was simply set as 3 times the mean GDP per capita in China during this study, which might have caused bias; thus, the acceptability analysis could be investigated further. Secondly, due to the imbalance of development between Southern and Northern China, as well as coastal areas and inland China, the cost-effectiveness threshold might be unaffordable in some relatively undeveloped regions. Therefore, further studies should be conducted to evaluate the cost-effectiveness of ABN + MTX treatment in the different regions of China. Thirdly, the sample size in this study was relatively small, and studies with larger sample sizes should be further conducted to verify our results. Finally, the follow-up duration of this study was relatively short, and the long-term cost-effectiveness of $\mathrm{ABN}+\mathrm{MTX}$ treatment in RA patients was unclear, which could also be investigated further.

\section{Conclusions}

Collectively, ABN + MTX treatment exhibits acceptable cost-effectiveness compared to cDMARDs treatment in Chinese RA patients; meanwhile, its cost-effectiveness presents some robustness to the turbulence of $A B N$ price and HAQ-DI score.

\section{Acknowledgments}

Funding: This study was supported by Provincial Natural Science Foundation of HuNan (2017JJ4069).

\section{Footnote}

Reporting Checklist: The authors have completed the CHEERS reporting checklist. Available at https://dx.doi. org/10.21037/atm-21-3132

Data Sharing Statement: Available at https://dx.doi. org/10.21037/atm-21-3132

Conflicts of Interest: All authors have completed the ICMJE uniform disclosure form (available at https://dx.doi. org/10.21037/atm-21-3132). The authors have no conflicts of interest to declare.

Ethical Statement: The authors are accountable for all aspects of the work in ensuring that questions related to the accuracy or integrity of any part of the work are appropriately investigated and resolved. This study was approved by the Ethics Committee of Zhuzhou Central Hospital (2015-K-05010). All procedures were conducted in accordance with the provisions of the Declaration of Helsinki (as revised in 2013) and Good Clinical Practice guidelines as defined by the International Conference on Harmonization. All participants provided written informed consent.

Open Access Statement: This is an Open Access article distributed in accordance with the Creative Commons Attribution-NonCommercial-NoDerivs 4.0 International License (CC BY-NC-ND 4.0), which permits the noncommercial replication and distribution of the article with the strict proviso that no changes or edits are made and the original work is properly cited (including links to both the formal publication through the relevant DOI and the license). See: https://creativecommons.org/licenses/by-nc-nd/4.0/.

\section{References}

1. Smolen JS, Aletaha D, Barton A, et al. Rheumatoid arthritis. Nat Rev Dis Primers 2018;4:18001.

2. Wang G, Mu R, Xu H. Management of rheumatoid arthritis in People's Republic of China - focus on tocilizumab and patient considerations. Int J Gen Med 
2015;8:187-94.

3. Aletaha D, Smolen JS. Diagnosis and Management of Rheumatoid Arthritis: A Review. JAMA 2018;320:1360-72.

4. Xie C, Jiang J, Liu J, et al. Ginkgolide B attenuates collagen-induced rheumatoid arthritis and regulates fibroblast-like synoviocytes-mediated apoptosis and inflammation. Ann Transl Med 2020;8:1497.

5. Fazal SA, Khan M, Nishi SE, et al. A Clinical Update and Global Economic Burden of Rheumatoid Arthritis. Endocr Metab Immune Disord Drug Targets 2018;18:98-109.

6. Smolen JS, Landewé R, Bijlsma J, et al. EULAR recommendations for the management of rheumatoid arthritis with synthetic and biological disease-modifying antirheumatic drugs: 2016 update. Ann Rheum Dis 2017;76:960-77.

7. Xiong H, Hu M, Huang H, et al. Eukaryotic translation initiation factor 3B downregulation inhibits cell proliferation and promotes cell apoptosis through negatively regulating tumor necrosis factor receptor superfamily member 21 in gastric cancer. Transl Cancer Res 2019;8:2242-51..

8. Kay J, Schoels MM, Dörner T, et al. Consensus-based recommendations for the use of biosimilars to treat rheumatological diseases. Ann Rheum Dis 2018;77:165-74.

9. Hu H, Luan L, Yang K, et al. Burden of rheumatoid arthritis from a societal perspective: A prevalence-based study on cost of this illness for patients in China. Int J Rheum Dis 2018;21:1572-80.

10. Chen XX, Dai Q, Huang AB, et al. A multicenter, randomized, double-blind clinical trial of combination therapy with Anbainuo, a novel recombinant human TNFRII:Fc fusion protein, plus methotrexate versus methotrexate alone or Anbainuo alone in Chinese patients with moderate to severe rheumatoid arthritis. Clin Rheumatol 2013;32:99-108.

11. Chen XX, Li ZG, Wu HX, et al. A randomized, controlled trial of efficacy and safety of Anbainuo, a bio-similar etanercept, for moderate to severe rheumatoid arthritis inadequately responding to methotrexate. Clin Rheumatol 2016;35:2175-83.

12. Tian F, Li JY, Wen ZH, et al. A novel etanercept biosimilar Anbainuo plus methotrexate exhibits increased costeffectiveness compared to conventional disease-modifying anti-rheumatic drugs in treating rheumatoid arthritis patients. Medicine (Baltimore) 2019;98:e17750.

13. Carreño A, Fernández I, Badia X, et al. Using HAQDI to estimate HUI-3 and EQ-5D utility values for patients with rheumatoid arthritis in Spain. Value Health
2011;14:192-200.

14. Kristensen LS, Andersen MS, Stagsted LVW, et al. The biogenesis, biology and characterization of circular RNAs. Nat Rev Genet 2019;20:675-91.

15. Spalding JR, Hay J. Cost effectiveness of tumour necrosis factor-alpha inhibitors as first-line agents in rheumatoid arthritis. Pharmacoeconomics 2006;24:1221-32.

16. Ashraf RBNST. UT3 ESTIMATING HEALTH UTILITY FROM A PHYSICAL FUNCTION ASSESSMENT IN RHEUMATOID ARTHRITIS (RA) PATIENTS TREATED WITH ADALIMUMAB (D2E7). Value in Health 2002;5:452-3.

17. Ducournau PKA, Wintfeld N. Comparison of linear and non-linear utility mapping between HAQ and EQ-5D using pooled data from the tocilizumab trials OPTION and LITHE. British Society of Rheumatology; Glasgow, 2009.

18. Bansback NJ, Brennan A, Ghatnekar O. Cost effectiveness of adalimumab in the treatment of patients with moderate to severe rheumatoid arthritis in Sweden. Ann Rheum Dis 2005;64:995-1002.

19. Burmester GR, Rigby WF, van Vollenhoven RF, et al. Tocilizumab in early progressive rheumatoid arthritis: FUNCTION, a randomised controlled trial. Ann Rheum Dis 2016;75:1081-91.

20. Huoponen S, Aaltonen KJ, Viikinkoski J, et al. Costeffectiveness of abatacept, tocilizumab and TNF-inhibitors compared with rituximab as second-line biologic drug in rheumatoid arthritis. PLoS One 2019;14:e0220142.

21. Gissel C, Götz G, Repp H. Cost-effectiveness of adalimumab for rheumatoid arthritis in Germany. Z Rheumatol 2016;75:1006-15.

22. Shi ZC, Fei HP, Wang ZL. Cost-effectiveness analysis of etanercept plus methotrexate vs triple therapy in treating Chinese rheumatoid arthritis patients. Medicine (Baltimore) 2020;99:e16635.

23. Kievit W, Maurits JS, Arts EE, et al. Cost-Effectiveness of Cardiovascular Screening in Patients With Rheumatoid Arthritis. Arthritis Care Res (Hoboken) 2017;69:175-82.

(English Language Editor: J. Jones)

Cite this article as: Tian F, Wen Z, Li J, Luo X, Deng L, Zhang L, He J, Yao F, Liao Z. Cost-effectiveness of Anbainuo plus methotrexate compared to conventional disease-modifying antirheumatic drugs for rheumatoid arthritis patients in China. Ann Transl Med 2021;9(14):1165. doi: 10.21037/atm-21-3132 


\section{Supplementary}

Table S1 Comparison of the cost-effectiveness using different utility formulas

\begin{tabular}{|c|c|c|c|c|c|}
\hline Utilities & QALY & Incremental QALY & Total cost $(¥)$ & Incremental cost $(¥)$ & ICER (¥/QALY) \\
\hline \multicolumn{6}{|c|}{$\mathrm{HUI}-3=0.9527-0.2018 \times \mathrm{HAQ}-\mathrm{DI}$} \\
\hline$A B N+M T X$ & 0.79 & 0.25 & $63,815.4$ & $32,516.8$ & $130,067.2^{*}$ \\
\hline Control & 0.64 & - & $31,298.6$ & - & \\
\hline \multicolumn{6}{|c|}{$\mathrm{HUI} 3=0.76+0.05 \times$ female $+0.001 \times$ age $-0.28 \times \mathrm{HAQ}-\mathrm{DI}$} \\
\hline $\mathrm{ABN}+\mathrm{MTX}$ & 0.64 & 0.21 & $63,815.4$ & $32,516.8$ & $154,841.9^{*}$ \\
\hline Control & 0.43 & - & $31,298.6$ & - & \\
\hline \multicolumn{6}{|c|}{ Using equation referring to the study [16] } \\
\hline \multicolumn{6}{|c|}{ QoL $=0.862-0.327 \times \mathrm{HAQ}-\mathrm{DI}$} \\
\hline Control & 0.36 & - & $31,298.6$ & - & \\
\hline \multicolumn{6}{|c|}{ Using equation referring to the study [17] } \\
\hline \multicolumn{6}{|c|}{$\mathrm{QoL}=0.8229-0.1125 \times \mathrm{HAQ}-\mathrm{DI}-0.06874 \times \mathrm{HAQ}-\mathrm{DI}^{2}$} \\
\hline$A B N+M T X$ & 0.68 & 0.21 & $63,815.4$ & $32,516.8$ & $154,841.9^{\star}$ \\
\hline Control & 0.47 & - & $31,298.6$ & - & \\
\hline \multicolumn{6}{|c|}{ Using equation referring to the study [18] } \\
\hline \multicolumn{6}{|c|}{ QoL $=0.76-0.28 \times$ HAQ-DI $+0.05 \times$ female } \\
\hline $\mathrm{ABN}+\mathrm{MTX}$ & 0.58 & 0.21 & $63,815.4$ & $32,516.8$ & $154,841.9^{*}$ \\
\hline
\end{tabular}

The average of gross domestic product (GDP) per capita in China from 2016 to 2019 was $¥ 62,634$. *, the ICER was less than 3 times of the mean GDP per capita. QALY, quality-adjusted life-years; ICER, incremental cost-effectiveness ratio; HUI3, health utilities index mark 3; HAQ-DI, Health Assessment Questionnaire Disability Index; ABN, anbainuo; MTX, methotrexate; QoL, quality of life. 